\title{
S-ASYMPTOTIC EXPANSION OF DISTRIBUTIONS
}

\author{
BOGOLJUB STANKOVIC \\ Institute of Mathematics \\ University of Nov1 Sad \\ Yugoslavia
}

(Received December 2, 1986 and in revised form Apri1 28, 1987)

ABSTRACT. This paper contains first a definition of the asymptotic expansion at infinity of distributions belonging to $\theta^{-}\left(\mathrm{R}^{\mathrm{n}}\right)$, named S-asymptotic expansion, as also its properties and application to partial differential equations.

KEYS WORDS AND PHRASES. Convex cone, distribution, behaviour of a distribution at infinity, asymptotic expansion.

1980 AMS SUBJECT CLASSIFICATION CODE. Primary 41A60, Secondary 46 F99.

1. INTRODUCTION.

The basic idea of the asymptotic behaviour at infinity of a distribution one can find already in the book of L. Schwartz [1]. To these days many mathematicians tried to find a good definition of the asymptotic behaviour of a distribution. We shall mention only "equivalence at infinity" explored by Lavoine and Misra [2] and the "quasiasymptotic" elaborated by vladimirov and his pupils [3]. Brichkov [4] introduced the asymptotic expansion of tempered distributions as a useful mathematical tool in quantum field theory. His investigations and definitions were turned just towards these applications. In [4] one can find cited literature in which asymptotic expansion technique, introduced by Brichkov, was used in the quantum field theory. This is a reason to study S-asymptotic expansion.

2. DEFINITION OF THE S-ASYMPTOTIC EXPANSION.

In the classical analysis we say that the sequence $\left\{\psi_{n}(t)\right\}$ of numerical functions is asymptotic if and only if $\psi_{n+1}(t)=o\left(\psi_{n}(t)\right), t \rightarrow \infty$. The formal series $\sum_{n \geq 1} u_{n}(t)$ is an asymptotic expansion of the function $u(t)$ related to the asymptotic sequence $\left\{\psi_{n}(t)\right\}$ if

$$
u(t)-\sum_{n=1}^{k} u_{n}(t)=o\left(\psi_{k}(t)\right), t \rightarrow \infty
$$

for every $k \in \mathrm{N}$ and we write

$$
u(t) \sim \sum_{n=1}^{\infty} u_{n}(t) \mid\left\{\psi_{n}(t)\right\}, t \rightarrow \infty
$$

When for every $n \in N \quad u_{n}(t)=c_{n} \psi_{n}(t), c_{n}$ are complex numbers, expansion (2.2) is unique, that means the numbers $c_{n}$ can be determined in only one way. 
In this text $\Gamma$ will be a convex cone with vertex at zero belonging to $R^{n}$ and $\Sigma(\Gamma)$ the set of all real valued and positive functions $c(h), h \in \Gamma$. Notations for the spaces of distributions are as in the books of Schwartz [1].

DEFINITION 1. The distribution $\mathrm{T} \in D^{-}$has the S-asymptotic expansion related to the asymptotic sequence $\left\{c_{n}(h)\right\} \subset \Sigma(\Gamma)$, we write it

$$
T(t+h) \stackrel{s}{\sum_{n=1}^{\infty}} U_{n}(t, h) \mid\left\{c_{n}(h)\right\},\|h\| \rightarrow \infty, h \in \Gamma
$$

where $U_{n}(t, h) \in D^{-}$for $n \in N$ and $h \in \Gamma$, if for every $\rho \in d \theta$

$$
<T(t+h), \rho(t)>\sim \sum_{n=4}^{\infty}<U_{n}(t, h), \rho(t)>\mid\left\{c_{n}(h)\right\},\|h\| \rightarrow \infty, h \in \Gamma
$$

REMARK. 1) In the special case $U_{n}(t, h)=u_{n}(t) c_{n}(h), u_{n} \in \mathscr{B}, n \in N$, we shall write

$$
T(t+h) \stackrel{s}{\sim} \sum_{n=1}^{\infty} u_{n}(t) c_{n}(h),\|h\|+\infty, h \epsilon
$$

and the given S-asymptotic expansion is unique.

2) To define the S-asymptotic expansion in $\mathscr{\mathscr { L }}^{\prime}\left(\mathrm{R}^{\mathrm{n}}\right)$, we have only to suppose that in relation (2.4) $\mathrm{T}$ and $\mathrm{U}_{\mathrm{n}}$ are in $\mathcal{L}$, and $\rho$ in $\mathcal{L}$.

Brichkov's general definition is slightly different [5].

DEFINITION $1^{\prime}$. The distribution $g \in \mathcal{L}^{\text {- }}$ has the asymptotic expansion related to the asymptotic sequence $\left\{\psi_{n}(t)\right\}$ on the ray $\left\{\lambda h_{0}, \lambda>0\right\}, h_{0} \in R^{n}$

$$
g\left(\lambda h_{0}-t\right) \sim \sum_{n=1}^{\infty} \hat{c}_{n}(t, \lambda) \mid\left\{\psi_{n}(\lambda)\right\}, \lambda \in R, \lambda \rightarrow \infty
$$

where $\hat{c}_{n}(t, \lambda) \in \mathcal{L}$, for $\lambda \geqq \lambda_{0}>0$, if for every $\phi \in \mathcal{\rho}$

$$
<g\left(\lambda h_{0}-t\right), \phi(t)>\sim \sum_{n=1}^{\infty}<\hat{c}_{n}(t, \lambda), \phi(t)>\mid\left\{\psi_{n}(\lambda)\right\}, \lambda \rightarrow \infty
$$

Relation 2.6 can be transformed in

$$
f(x) e^{i \lambda \ll x, h_{0} \gg} \sim \sum_{n=1}^{\infty} c_{n}(x, \lambda) \mid\left\{\psi_{n}(\lambda)\right\}, \lambda \rightarrow \infty
$$

by the Fourier transform, if we take $f(x)=F^{-1}[g(t)] ; \rho(x)=F^{-1}[\phi(t)]$ and $F\left[\hat{c}_{n}(t, \lambda)\right]=(2 \pi)^{n} c_{n}(x, \lambda)$. We denote by $F[\rho]$ the Fourier transform of $\rho$ and by $F^{-1}[g]$ the inverse Fourier transform of $g$. Also, for $x, t \in R^{n} \ll x, t \gg=\sum_{i=1}^{n} x_{i} t_{i}$.

In his papers Bric̀hkov considered only the asymptotic expansinons (2.8) and in one dimensional case. We shall study the asymptotic expansion not in $f^{\prime}(\mathrm{R})$ but in the whole $D^{-}\left(R^{n}\right)$, not only on a ray but on a cone in $R^{n}$. Our results enlarge Brichkov's to be valued for the elements of $D^{-}\left(R^{n}\right)$ (Corollary 1), they are proved with less suppositions (Propositions 5 and 6) or give new properties of the S-asymptotic.

A distribution belonging to $\mathcal{D}^{-}$can have S-asymptotic expansion in $D^{-}$without having the same S-asymptotic expansion in $\mathcal{L}$. Such an example is the regular distribution $\tilde{\mathrm{f}}$ defined by the function

$$
f(t)=H(t) \exp \left(1 /\left(1+t^{2}\right)\right) \exp (-t), \quad t \in R
$$


where

$$
H(t)=1, t \geqq 0 \text { and } H(t)=0, \quad t<0 \text {. }
$$

It is easy to prove that for $h \in R_{+}$

$$
\tilde{\mathrm{f}}(\mathrm{t}+\mathrm{h}) \stackrel{\mathrm{\sim}}{\sum_{\mathrm{n}=1}^{\infty}} \frac{1}{(n-1) !}\left(1+(t+h)^{2}\right)^{1-n} \exp (-t-h) \mid\left\{\mathrm{e}^{-h_{h} 2(1-n)}\right\}, h \rightarrow \infty \text {. }
$$

But

$$
U_{n}(t, h)=\left(1+(t+h)^{2}\right)^{1-n} \exp (-t-h), \quad n \in N, \quad h>0
$$

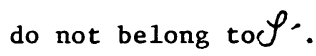

The regular distribution $\tilde{\mathrm{g}}$ defined by the function

$$
g(t)=\exp \left(1+\left(1+t^{2}\right)\right) \exp (t), \quad t \in R
$$

belongs to $\mathcal{D}^{-}$but it is not in $\mathcal{S}^{\prime}$. It has s-asymptotic expansion in $\mathscr{D}^{-}$:

$$
\tilde{g}(t+h) \stackrel{s}{\sum_{n=1}^{\infty}} \frac{1}{(n-1) !}\left(1+(t+h)^{2}\right)^{1-n} \exp (t+h) \mid\left\{e^{h h^{2(1-n)}}\right\}, h \rightarrow \infty
$$

where $\mathrm{l}^{\mathrm{N}}=\mathrm{R}_{+}$.

3. PROPERTIES OF THE S-ASYMPTOTIC EXPANSION.

PROPOSITION 1. Let $S \in \mathcal{E}^{\prime}$ and $T \in \mathscr{D}^{\prime}$. If

$$
T(t+h) \stackrel{s}{\sum_{n=1}^{\infty}} U_{n}(t, h) \mid\left\{c_{n}(h)\right\},\|h\| \rightarrow \infty, h \in \Gamma
$$

then the convolution

$$
(S * T)(t+h) \stackrel{s}{\sim} \sum_{n=1}^{\infty}\left(S * U_{n}\right)(t, h) \mid\left\{c_{n}(h)\right\} \quad\|h\| \rightarrow \infty h \in \Gamma
$$

PROOF. We know that

$$
\langle(S * T)(t+h), \rho(t)\rangle-\sum_{n=1}^{k}\left\langle\left(S * U_{n}\right)(t, h), \rho(t)\right\rangle=\left\langle S *\left[T(t+h)-\sum_{n=1}^{k} U_{n}(t, h)\right], \rho(t)\right\rangle .
$$

It remains only to use the continuity of the convolution.

COROLLARY 1. If

$$
T(t+h) \stackrel{s}{\sim} \sum_{n=1}^{\infty} U_{n}(t, h) \mid\left\{c_{n}(h)\right\} \quad\|h\| \rightarrow \infty, h \in \Gamma
$$

then

$$
\begin{aligned}
& T^{(k)}(t+h) \stackrel{s}{s} \sum_{n=1}^{\infty} U_{n}^{(k)}(t, h) \mid\left\{c_{n}(h)\right\},\|h\| \rightarrow \infty, h \in \Gamma \\
& \text { where } T^{(k)}=\left(D_{t_{1}}^{k_{1}} \ldots D_{t_{n}}^{k_{n}^{n}}\right) T, k=\left(k_{1}, \ldots, k_{n}\right) \in N_{0}^{n}, N_{0}=N U\{0\} \text {. }
\end{aligned}
$$

PROOF. We have only to take $S=\delta^{(k)}$ in Proposition 1.

REMARK. Proposition 1 . is valued as well if we suppose that $\mathrm{T} \epsilon \mathcal{\rho}^{\prime}$ and $\mathrm{s} \epsilon O_{\mathrm{c}}$. PROPOSITION 2. Let $f, U_{n}(t, h)$ and $v_{n}(t), n \in N$ and $h \in \Gamma$, be the local integrable functions such that for every compact set $K \subset R^{n}$

$$
f(t+h) \sim \sum_{n=1}^{\infty} U_{n}(t, h) \mid\left\{c_{n}(h)\right\},\|h\| \rightarrow \infty, h \in \Gamma, t \in K
$$


and

$$
\left|f(t+h)-\sum_{n=1}^{k} U_{n}(t, h)\right| / c_{k}(h) \leqq v_{k}(t), t \in K, h \in \Gamma
$$

and $\|h\| \geqq r(k, K)$, then for the regular distribution $\tilde{f}$ defined by $f$ we have

$$
\tilde{\mathrm{f}}(\mathrm{t}+\mathrm{h}) \stackrel{s}{\sim} \sum_{n=1}^{\infty} \tilde{\mathrm{U}}_{\mathrm{n}}(t, h) \mid\left\{c_{n}(h)\right\},\|\mathrm{h}\| \rightarrow \infty, \mathrm{h} \in \Gamma .
$$

PROOF. The proof is a consequence of the Lebesgue's theorem.

PROPOSITION 3. Suppose that $T_{1}$ and $T_{2}$ belong to $d^{-}$and equal over the open set $\Omega$ which has the property: for every $r>0$ there exists a $\beta_{0}$ such that the ball $B(0, r)=\left\{x \in R^{n},\|x\| \leqq r\right\}$ is in $\left\{\Omega-h, h \in \Gamma,\|h\| \geq \beta_{o}\right\}$. If

$$
T_{1}(t+h) \underbrace{s}_{n=1} U_{n}(t, h) \mid\left\{c_{n}(h)\right\},\|h\| \rightarrow \infty, h \in r
$$

then

$$
T_{2}(t+h) s \sum_{n=1}^{\infty} U_{n}(t, h) \mid\left\{c_{n}(h)\right\},\|h\| \rightarrow \infty, h \in \Gamma
$$

as we11.

PROOF. We have only to prove that for every $c_{k}(h)$

$$
\| \lim _{h \|+\infty, h \in \Gamma}<\left[T_{1}(t+h)-T_{2}(t+h)\right] / c_{k}(h), \rho(t)>=0, \rho \in D
$$

Let supp $\rho \subset B(0, r)$. The distribution $T_{1}(t+h)-T_{2}(t+h)$ equals zero over $\Omega-h$. By the supposition there exists a $\beta_{0}$ such that the ball $B(0, r)$ is in $\{\Omega-h, h \in \Gamma$, $\left.\|h\| \geq \beta_{0}\right\}$. This proves out relation (3.3).

PROPOSITION 4. Let $S \in \mathbb{D}^{-}$and for $1 \leqq m \leqq n$

$$
D_{t_{m}} S(t+h) s \sum_{i=1}^{\infty} U_{1}(t, h) \mid\left\{c_{1}(h)\right\},\|h\| \rightarrow \infty, h \in \Gamma .
$$

If the family $\left\{v_{i}(t, h), i \in N, h \in \Gamma\right\}$ has the properties: $D_{t_{m}} v_{1}(t, h)=U_{i}(t, h)$, $1 \in N, h \in \Gamma$ and for $a \rho_{0} \in D(R), \int_{R} \rho_{0}(\tau) d \tau=1$, and for every $\rho^{m} \in \mathcal{L}, k \in N$

$$
\|\mathrm{h}\| \lim _{1 \rightarrow \infty, h \in \Gamma}<\left[S(t+h)-\sum_{i=4}^{k} v_{i}(t, h)\right] / c_{k}(h), \rho_{0}\left(t_{m}\right) \lambda_{m}(t)>=0
$$

where $\lambda_{m}(t)=\int_{R} \rho\left(t_{1}, \ldots, t_{m}, \ldots, t_{n}\right) d t_{m}$, then

$$
S(t+h) s \sum_{i=1}^{\infty} v_{i}(t, h) \mid\left\{c_{1}(h)\right\},\|h\| \rightarrow \infty, h \in \Gamma .
$$

PROOF. If $\rho \in \mathcal{D}$ then $\rho(t)=\rho_{0}\left(t_{m}\right) \lambda_{m}(t)+\psi(t)$ where $\psi \in D$ and $\int_{R} \psi\left(t_{1}, \ldots, t_{m}, \ldots, t_{n}\right) d t_{m}=0$.

Now we have the following equality

$$
\begin{aligned}
\langle[S(t+h)- & \left.\left.-\sum_{i=1}^{k} v_{i}(t, h)\right], \rho(t)\right\rangle=\left\langle\left[S(t+h)-\sum_{i=1}^{k} v_{i}(t, h)\right], \rho_{o} \lambda_{m}(t)\right\rangle \\
& -\left\langle\left[D_{t_{m}} S(t+h)-\sum_{i=1}^{k} U_{i}(t+h)\right], \int_{-\infty}^{t m} \psi\left(t_{1}, \ldots, u_{m}, \ldots, t_{n}\right) d u_{m}\right\rangle .
\end{aligned}
$$

It remains only to use the limit in it and Corollary 1. 
PROPOSITION 5. Suppose that $S \in \theta^{-}, \Gamma=\left\{h \in R^{n}, h=\left(0, \ldots, h_{m}, \ldots, 0\right)\right\}$, where $\mathrm{m}$ is fixed, $1 \leqq \mathrm{~m} \leqq \mathrm{n}$ and

$$
\left(D_{t} S\right)(t+h) s \sum_{i=1}^{\infty} U_{i}(t, h) \mid\left\{c_{i}(h)\right\},\|h\| \rightarrow \infty, h \in \Gamma
$$

If there exists $V_{i}(t, h), D_{h_{m}} V_{i}(t, h)=U_{i}(t, h), i \in N$ and if $c_{i}(h), i \in N$ are local integrable in $\mathrm{h}_{\mathrm{m}}$ and such that

$$
\hat{c}_{i}(h)=\int_{1}^{h_{m}} c_{i}(u) d u_{m} \rightarrow \infty \text { as } h_{m} \rightarrow \infty
$$

then

$$
S(t+h) \stackrel{s}{\sim} \sum_{i=1}^{\infty} v_{i}(t, h) \mid\left\{\hat{c}_{i}(h)\right\},\|h\| \rightarrow \infty, h \in \Gamma .
$$

PROOF. By L'Hospital's rule with the Stolz's improvement we have for every $\rho \in \mathcal{D}$ and $k \in N$

$$
\begin{aligned}
& \text { and } k \in N \\
& \lim _{h \rightarrow \infty, h \in \Gamma} \frac{\langle S(t+h), \rho(t)\rangle-\left\langle\sum_{i=1}^{k} v_{i}(t, h), \rho(t)\right\rangle}{\hat{c}_{k}(h)} \\
& =\lim _{h \rightarrow \infty, h \in \Gamma} \frac{\left.\left\langle\left(D_{t_{m}} S\right)(t+h), \rho(t)\right\rangle-<\sum_{i=1}^{k} U_{i}(t, h), \rho(t)\right\rangle}{c_{k}(h)} .
\end{aligned}
$$

These five propositions give how is related the S-asymptotic with convolution, derivative, classical expansion and the primitive of a distribution. The next proposition gives the analytical expression of $U_{n}(t, h)=u_{n}(t) c_{n}(h)$.

PROPOSITION 6. Suppose that $T \in \mathcal{D}^{-}$, $\Gamma^{\prime}$ with nonempty interior,

$$
T(t+h) \stackrel{s}{\sum_{n=1}^{\infty}} u_{n}(t) c_{n}(h),\|h\| \rightarrow \infty, h \in \Gamma .
$$

If $u_{m} \neq 0, m \in N$, then $u_{m}$ has the form

$$
u_{m}(t)=\sum_{k=1}^{m} P_{k}^{m}\left(t_{1}, \ldots, t_{n}\right) \exp \left(\ll a^{k}, t \gg\right), m \in N
$$

where $a^{k}=\left(a_{1}^{k}, \ldots, a_{n}^{k}\right) \in R^{n}$ and $P_{k}^{m}$ are polynomials, the power of them less of $k$ in every $t_{i}, i=1, \ldots, n: \ll x, t \gg=\sum_{1=1}^{n} x_{i} t_{i}$.

PROOF. By Definition 1 and our supposition

$$
\lim _{\|h\| \rightarrow \infty, h \in \Gamma} T(t+h) / c_{1}(h)=u_{1}(t) \neq 0
$$

From relation (3.5) follows that $u_{1}$ satisfies the equation

$$
u_{1}\left(t+h_{0}\right)=d\left(h_{0}\right) u_{1}(t), h_{0} \in \Gamma
$$

where

$$
d\left(h_{0}\right)=\lim _{\|h\| \rightarrow \infty, h \in \Gamma} c_{1}\left(h+h_{0}\right) / c_{1}(h)
$$


If $h_{o}$ is an interior point of $\Gamma$ and $e_{k}$ is such element from $R^{n}$ for which all the coordinates equal zero except the $k$-th which is 1 . Then

$$
u_{1}\left(t+h_{0}+\varepsilon e_{k}\right)-u_{1}\left(t+h_{0}\right)=\left[d\left(\varepsilon e_{k}\right)-d(0)\right] u_{1}\left(t+h_{0}\right)
$$

Hence the existence of $D_{h_{k}} d(h)_{h=0}=a_{k}^{1}$ and

$$
D_{t_{k}} u_{1}\left(t+h_{0}\right)=a_{k}^{1} u_{1}\left(t+h_{o}\right), k=1, \ldots, n \text {. }
$$

We know that all the solutions of equation (3.7) are of the form $u_{1}(t)=C_{1} \exp (\ll a, t \gg)$, where $C_{1}$ is a constant and $a^{1}=\left(a_{1}^{1}, \ldots, a_{n}^{1}\right)$.

The following limit gives $\mathrm{u}_{2}$

$$
\lim _{\|h\| \rightarrow \infty, h \in \Gamma} \frac{\langle T(t+h), \rho(t)\rangle-\left\langle u_{1}(t), \rho(t)\right\rangle c_{1}(h)}{c_{2}(h)}=\left\langle u_{2}, \rho\right\rangle
$$

By Corollary 1 follows for $1=1, \ldots, n$

$$
\lim _{\|h\| \rightarrow \infty, h \in \Gamma} \frac{\left\langle\left(D_{t_{i}}-a_{i}^{1}\right) T(t+h), \rho(t)\right\rangle}{c_{2}(h)}=\left\langle\left(D_{t_{i}}-a_{i}^{1}\right) u_{2}(t), \rho(t)\right\rangle
$$

Two cases are possible. a) If $\left(D_{t_{i}}-a_{1}^{1}\right) u_{2}=0, i=1, \ldots, n$, then $u_{2}(t)=C_{2} \exp \left(\ll a^{1}, t \gg\right)$.

b) If $\left(D_{t_{i}}-a_{i}^{1}\right) u_{2} \neq 0$ for some 1 , then $\left(D_{t_{i}}-a_{i}^{1}\right) u_{2}(t)=c \exp \left(\ll a^{2}, t \gg\right)$ and $u_{2}$ has the form $C_{2} \exp \left(\ll a^{1}, t \gg\right)+P_{2}^{2}\left(t_{1}, \ldots, t_{n}\right) \exp \left(\ll a^{2}, t \gg\right)$, where $P_{2}^{2}$ is a polynomial of the power less of 2 in every $t_{1}, i=1, \ldots, n$.

In the same way we prove for every $u_{m}$.

PROPOSITION 7. Let $T \in D^{-}$and $\Omega \in R^{n^{m}}$ be an open set with the property: for every $r>0$ there exists a $\beta_{r}$ such that the ball $B(h, r) \subset \Omega$ for all he $\Gamma,\|h\| \geq \beta_{r}$. Suppose

$$
T(t+h) \stackrel{s}{s} \sum_{n=1}^{m} U_{n}(t+h) \mid\left\{c_{1}(h), \ldots, c_{m}(h)\right\},\|h\| \rightarrow \infty, h \in \Gamma
$$

for any function $c_{m}(h)$ from $\Sigma(\Gamma)$, then $T=\sum_{n=1}^{m} U_{n}$ over $\Omega$.

PROOF. The statement of this Proposition can be obtained from a proposition proved in [6]. However, for completeness, we shall give the proof on the whole.

First we shall prove that if for every $c_{m}(h) \in \Sigma(\Gamma)$

$$
\lim _{\|h\|+\infty, h \in \Gamma}<\frac{T(t+h)-\sum_{n=1}^{m} U_{n}(t+h)}{c_{m}(h)}, \rho(t)>=0
$$

then there exists a $\beta(\rho)$ such that

$$
<\left[T(t+h)-\sum_{n=1}^{m} U_{n}(t+h)\right], \rho(t)>=0, h \in \Gamma,\|h\| \geqq \beta(\rho) .
$$

Suppose the opposite. We would have a sequence $h_{n} \in \Gamma,\left\|h_{n}\right\| \rightarrow \infty$ such that

$$
<\left[T\left(t+h_{n}\right)-\sum_{n=1}^{m} U_{n}\left(t+h_{n}\right)\right], \rho(t)>=p_{n} \neq 0, n \in N
$$

then we choose $c_{m}(h)$ in such a way that $c_{m}\left(h_{n}\right)=p_{n}$ and relation (3.8) would be false. 
We denote by $\beta_{0}(\rho)=\inf \beta(\rho)$. We shall prove that the set $\left\{\beta_{0}(\rho), \rho \in \theta_{K}\right\}$ for every compact set $K \subset R^{n}$ is bounded. Let us suppose the opposite; then there exists a sequence $\left\{h_{k}\right\}, h_{k} \in \dot{\Gamma},\left\|h_{k}\right\| \rightarrow \infty$ and the sequence $\left\{\phi_{k}(t)\right\}, \phi_{k} \in \mathcal{Q}_{k}$ such that

$$
\left\langle\overline{\mathrm{T}}\left(\mathrm{t}+\mathrm{h}_{\mathrm{k}}\right), \phi_{\mathrm{p}}(\mathrm{t})>=\mathrm{A}_{\mathrm{k}, \mathrm{p}}=\left\{\begin{array}{ll}
\mathrm{a}_{\mathrm{k}} \neq 0, & \mathrm{p}=\mathrm{k} \\
0, & \mathrm{p}<\mathrm{k}
\end{array} ; \overline{\mathrm{T}}=\mathrm{T}-\sum_{\mathrm{n}=1}^{\mathrm{m}} \mathrm{U}_{\mathrm{n}}\right. \text {. }\right.
$$

The construction of the sequence $\left\{\mathrm{h}_{\mathrm{k}}\right\}$ and $\phi_{\mathrm{k}}$ can be the following. Let $\phi_{\mathrm{k}} d_{\mathrm{K}}$ be such that $\beta_{0}\left(\phi_{k}\right)$ is a strict monotone sequence which tends to infinity, then there exist $\left\{h_{k}\right\} \subset \Gamma$ and $\varepsilon_{k}>0, k \in N$ such that $\beta_{0}\left(\phi_{k-1}\right)+\varepsilon_{k} \leqq\left\|h_{k}\right\| \leqq \beta_{0}\left(\phi_{k}\right)-\varepsilon_{k}$. Now, we shall construct the sequence $\left\{\psi_{\mathrm{p}}(t)\right\}, \psi_{\mathrm{p}} \in \mathcal{\theta}_{\mathrm{K}}$ for which we have

$$
\left\langle\bar{T}\left(t+h_{k}\right), \psi_{p}(t)>=\left\{\begin{array}{ll}
0, & p \neq k \\
a_{k}, & p=k
\end{array} .\right.\right.
$$

Let $\psi_{p}(t)=\phi_{p}(t)-\lambda_{1}^{p_{\phi}}(t)-\ldots-\lambda_{p-1}^{p} \phi_{p-1}(t), p>1$. The numbers $\lambda_{1}^{p}$ we can find in such a way that $\psi_{p}(t)$ satisfies the sought property.

It is easy to see that $\left\langle\overline{\mathrm{T}}\left(t+h_{k}\right), \psi_{k}(t)\right\rangle=a_{k}$ and $\left.\left\langle\bar{T}\left(t+h_{k}\right), \psi_{p}(t)\right\rangle=0, k\right\rangle$. For a fixed $p$ and $k<p$ we can find $\lambda_{i}^{p}, i=1, \ldots, p-1$ so that for $k=1, \ldots, p-1$

$$
0=\left\langle\bar{T}\left(t+h_{k}\right), \psi_{p}(t)\right\rangle=A_{k, p}-\lambda_{1}^{p} A_{k, 1}-\ldots-\lambda_{p-1}^{p} A_{k, p-1}
$$

Hence

$$
\lambda_{1}^{p} A_{k, 1}+\ldots+\lambda_{p-1}^{p} A_{k, p-1}=A_{k, p}, k=1, \ldots, p-1, p>1
$$

As $A_{k, k} \neq 0$ for every $k$, this system has always a solution.

We introduce now a sequence of numbers $\left\{b_{k}\right\}, b_{k}=\sup \left\{2^{k}\left|\psi_{k}^{(i)}(t)\right|, i<k\right\}$.

Then the function

$$
\psi(t)=\sum_{\mathrm{p}=1}^{\infty} \psi_{\mathrm{p}}(\mathrm{t}) / \mathrm{b}_{\mathrm{p}} \in D_{\mathrm{K}}
$$

and this series converges in $P_{K}$, thus in $D$ as well. With this

$$
\left\langle\bar{T}\left(t+h_{k}\right), \psi(t)\right\rangle=\sum_{p=1}^{\infty}\left\langle\bar{T}\left(t+h_{k}\right), \psi_{p}(t) / b_{p}\right\rangle=a_{k} / b_{k}
$$

If we choose $c(h)$ such that $c\left(h_{k}\right)=a_{k} / b_{k}$ then $\langle[\bar{T}(t+h) / c(h)], \psi(t)\rangle$ does not converge to zero when $\|h\| \rightarrow \infty, h \in \Gamma$. This is in contradiction with (3.8). Hence, for every compact set $K$ there exists a $\beta_{0}(K)$ such that $\langle\bar{T}(t+h), \phi(t)\rangle=0,\|h\| \geqq \beta_{0}(K)$, $h \in \Gamma, \phi \in \theta_{\mathrm{K}}$. That means that $\overline{\mathrm{T}}(\mathrm{t}+\mathrm{h})=0$ over $\mathrm{B}(0, \mathrm{r}),\|\mathrm{h}\| \geqq \beta(\mathrm{r}), \mathrm{h} \epsilon \Gamma$ and $\bar{T}(t)=0$ over $B(h, r),\|h\| \geqq B(r), h \in \Gamma$.

4. APPLICATION OF THE S-ASYMPTOTIC EXPANSION TO PARTIAL DIFFERENTIAL EQUATIONS.

As we mentioned in [4], one can find cited literature in which asymptotic expansion technique (in $\mathcal{L}^{-}$and in one dimensional case) was used in the quantum field theory. We show how the S-asymptotic expansion in $\mathscr{D}^{-}$can be applied to solutions of partial differential equations.

PROPOSITION 8. Suppose that $\mathrm{E}$ is a fundamental solution of the operator

$$
L(D)=\sum_{|\alpha| \geqq 0} a_{\alpha} D^{\alpha}, a_{\alpha} \in R, \alpha \in(N \cup 0)^{n} ; L(D) \not \equiv 0
$$

such that 


$$
E(t+h) \stackrel{s}{\infty} \sum_{n=1}^{\infty} u_{n}(t, h) \mid\left\{c_{n}(h)\right\},\|h\| \rightarrow \infty, h \in \Gamma .
$$

Then there exists a solution $x$ of the equation

$$
L(D) X=G, G \in \mathcal{E}^{-}
$$

which has S-asymptotic expansion

$$
X(t+h) \leqslant \sum_{n=1}^{\infty}\left(G * u_{n}(t, h)\right) \mid\left\{c_{n}(h)\right\},\|h\| \rightarrow \infty, h \in \Gamma .
$$

PROOF. The wel1-known Malgrange-Ehrenpreis theorem (see for example [7], p. 212) asserts that there exists a fundamental solution of the operator (4.1) belonging to D. The solution of equation (4.3) exists and can be expressed by the formula $\mathrm{X}=\mathrm{E} * \mathrm{G}$. To find the S-asymptotic of $\mathrm{X}$ we have only to apply Propostition 1 .

REMARKS. If we denote by $A(L(D), E)$ the collection of those $T \in D^{-}$for which the convolution $\mathrm{E} * \mathrm{~T}$ and $\mathrm{L}(\mathrm{D}) \delta * \mathrm{E} * \mathrm{~T}$ exist in $\mathcal{D}^{\prime}$, then the solution $\mathrm{X}=\mathrm{E} * \mathrm{G}$ is unique in the class $A(L(D), E)([7], p .87)$.

We can enlarge the space to which belongs $G([7]$, p. 216).

The fundamental solutions are known for the most important operators $L(D)$. ACKNOWLEDGEMENT. This material is based on work supported by the U.S.-Yugoslavia Joint Fund for Scientific and Technological Cooperation, in cooperation with the NSF under Grant (JFP) 544.

\section{REFERENCES}

1. SCHWARTZ, L. Théorie des distributions, T. I, II, Herman, Paris, 1957-1951.

2. LAVOINE, J. and MISRA, O.P. Théorèmes Abélians pour la transformation de Stieltjes des distributions, C.R. Acad. Sci. Paris Sér. I Math. T. 279 (1974), 99-102.

3. VLADIMIROV, v.S., DROŽŽINOV, Yu.N., and ZAVJALOV, B.I. Multidimensional Theorems of Tauberian Type for Generalized Functions, Moskva, "Nauka", 1986.

4. BRICHKOV, Yu.A. Asymptotical Expansions of the Generalized Functions I, Theoret. Mat. Fiz. T. $\underline{5}$ (1970), 98-109.

5. ZEMANIAN, A.H. Generalized Integral Transformations, Appendix $I$ in the Russian translation, Moskva "Nauka", 1974.

6. STANKOVIĆ, B. Characterization of Some Subspaces of $\infty^{\circ}$ by S-asymptotic, Publ. Inst. Mat. Beograd 41 (1987), in print.

7. VLADIMIROV, V.s. Generalized Functions in Mathematical Physics, Mir Publishers, Moscow, 1979. 


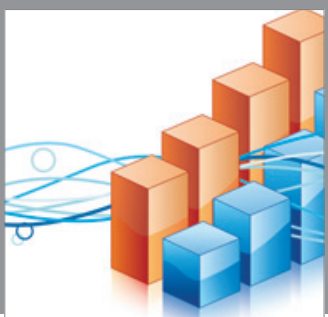

Advances in

Operations Research

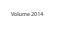

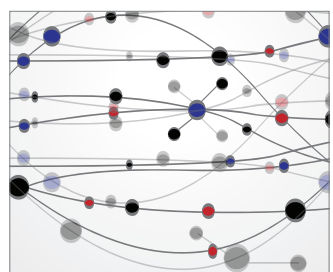

\section{The Scientific} World Journal
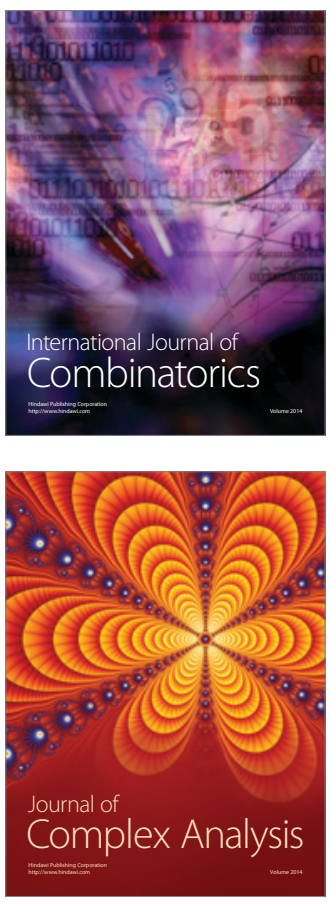

International Journal of

Mathematics and

Mathematical

Sciences
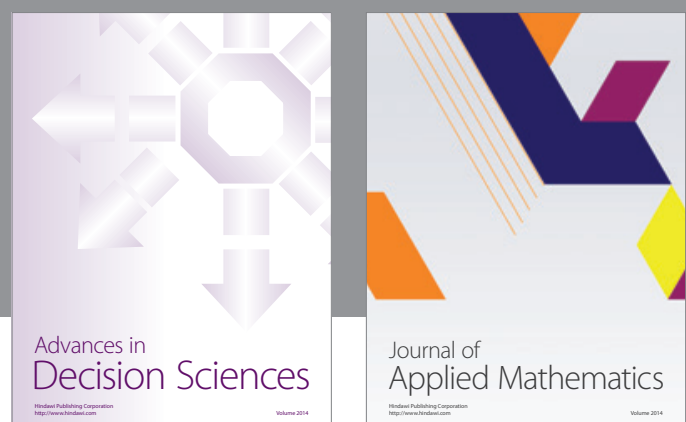

Journal of

Applied Mathematics
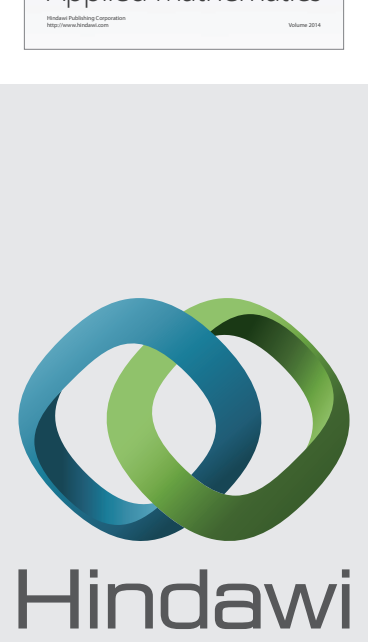

Submit your manuscripts at http://www.hindawi.com
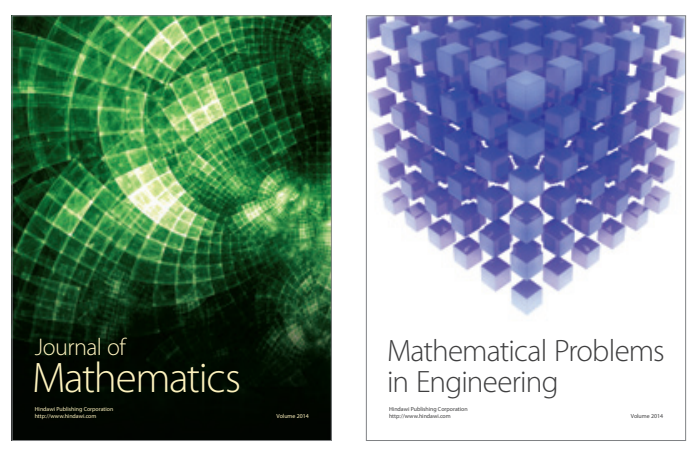

Mathematical Problems in Engineering
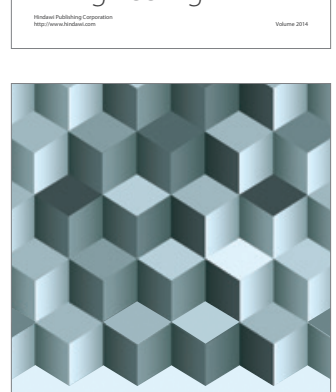

Journal of

Function Spaces
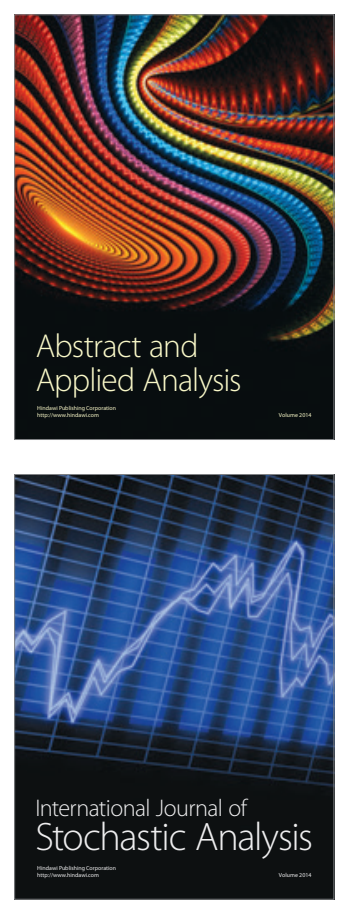

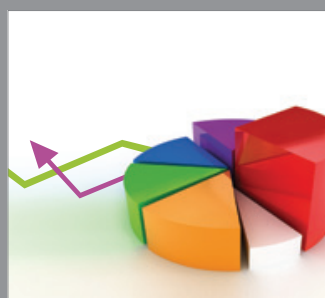

ournal of

Probability and Statistics

Promensencen
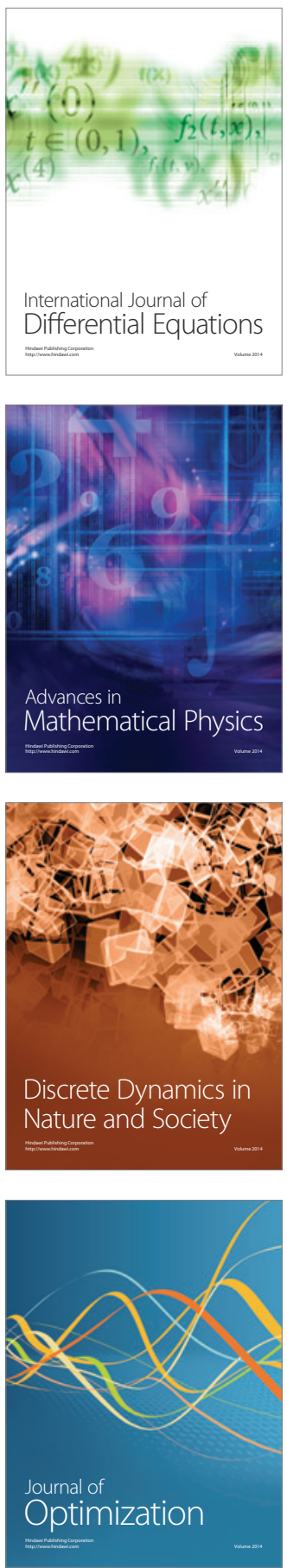